(c) 2018 IEEE. Personal use of this material is permitted. Permission from IEEE must be obtained for all other uses, in any current or future media, including reprinting/republishing this material for advertising or promotional purposes, creating new collective works, for resale or redistribution to servers or lists, or reuse of any copyrighted component of this work in other works.

Digital Object Identifier (DOI): 10.1109/TIA.2016.2581763

IEEE Transactions on Industry Applications (Volume: 52, Issue: 5, Sept. 2016)

H8 Inverter for Common-Mode Voltage Reduction in Electric Drives

Luca Concari

Davide Barater

Giampaolo Buticchi

Carlo Concari

Marco Liserre

Suggested Citation

L. Concari, D. Barater, G. Buticchi, C. Concari and M. Liserre, "H8 Inverter for Common-Mode Voltage Reduction in Electric Drives," in IEEE Transactions on Industry Applications, vol. 52, no. 5, pp. 40104019, Sept.-Oct. 2016. 


\title{
H8 Inverter for Common-Mode Voltage reduction in Electric Drives
}

\author{
Luca Concari, Graduate Student Member, IEEE, Davide Barater, Member, IEEE, Giampaolo Buticchi, Member, \\ IEEE, Carlo Concari, Member, IEEE, and Marco Liserre, Fellow, IEEE
}

\begin{abstract}
This paper presents a modified two-level three-phase inverter for the reduction of the leakage current. With respect to a traditional two-level inverter, the proposed solution reduces the common-mode voltage, both in amplitude and frequency. Between the DC source and the traditional three-phase bridge, two active DC-decoupling devices and a voltage-clamping network have been added. A dedicated control strategy was developed adopting a modified Space Vector PWM modulation, oriented to the reduction of the common-mode voltage. Simulations showing the good performance of the solution are presented. A preliminary prototype was developed and experimental results are presented.
\end{abstract}

Index Terms-Three-phase, inverter, drive, H8, PWM, space vector, common-mode voltage, leakage current.

\section{INTRODUCTION}

Despite the fact that the two-level three-phase inverters are widely used in electronics, it is well known that they exhibit poor common-mode voltage (CMV) characteristics. The existence of several common mode impedance paths between the converter and the motor/drive frame allows leakage currents to flow at every CMV variation, so that high pulse-width modulation frequencies produce high common-mode leakage currents [1]. In motor drive applications, this may lead to motor bearing failures, EMI noise that causes inverter drive trip, or interference with other electronic equipment in the vicinity [2].

The leakage current problem can be faced by reducing as much as possible the CMV, both in amplitude and frequency; ultimately, a constant CMV would not produce such leakage currents. Passive solutions, adopting passive filters at the inverter output or input, end up with increased system cost and size [3]. Depending on the application field, one aspect or the other may represent a more significant drawback. For example, size and weight are particularly relevant in aircraft applications [4], while cost still is significant in more traditional, industrialbased applications. Furthermore, since these currents flow through the system enclosure, they are even more relevant in case of carbon fiber structure used in modern aircraft applications.

This paper proposes a modified two-level inverter to solve the problem. It is an upgraded version of [5], where the same solution was originally presented. Section II presents a brief state of the art review of converter architectures and modulation strategies with reduced CMV. In section III a novel solution is proposed, which takes advantage both from a new topology and from a dedicated control strategy to reduce the CMV.

\section{STATE OF THE ART}

In the past, several authors dealt with the CMV problem adopting specific control strategies. In [6] and [7] several PWM strategies are investigated, but they suffer from drawbacks like not allowing to completely eliminate the CMV, leading to high current ripple or distortion or even being not practically feasible. In [8] a combined algorithm with satisfactory CMV reduction performance is proposed, but it still exhibits CMV over the PWM period, although reduced in amplitude and frequency compared to a traditional PWM modulation. For systems including a rectifier and an inverter, the CMV excursion can be reduced taking into account the supply AC voltage waveform for the definition of the SV pattern [9]. In presence of a fully controllable back-to-back rectifier/inverter configuration, synchronizing the rectifier and inverter PWM sequence allows to reduce the number of CMV pulses within a PWM period [10].

In addition to the traditional three-phase bridge (H6), different architectures have been proposed during the past years. Here a list of the most relevant ones is presented.

- The Neutral Point Clamped (NPC) inverter, shown in Fig. 1, was proposed for the first time in [11]; since then, several publications have focused on this topology, which offers benefits such as reduced switching losses, small output current ripple, and split supply voltage. Its main drawback consist in the complexity: more devices and more driver circuitry are necessary compared to other architectures and the control turns out to be more complex as well.

- Another approach to the CMV-related problems consist in a four-legs inverter like that one in Fig. 2, where a fourth leg is added to the H6 for controlling the CMV. Adopting a specific modulation, it can reduce the average output voltage and the differential mode distortion [12].

Other than in motor drive applications, CMV and common mode current are relevant in PV generation adopting transformerless conversion. As a matter of fact, there is a common mode path which includes the panels, the inverter, the grid, the ground and the stray capacitance between the panels and their supporting structure. In this context several modulation techniques have been studied [18] and alternative topologies proposed with the same aim of reducing the CMV and the leakage current.

- The topology proposed in [13] and represented in Fig. 3 reduces the CMV and the leakage current, but it has a great number of components (diodes and switches) which 


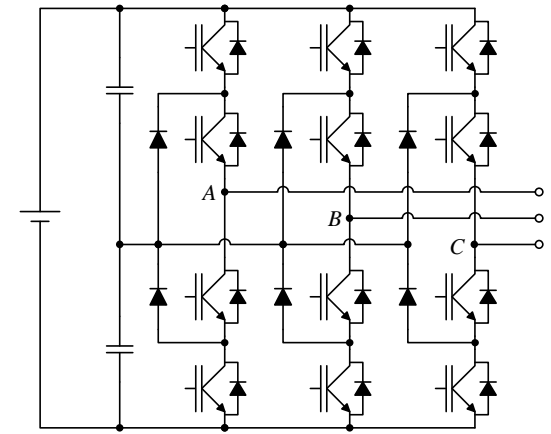

Figure 1. The Neutral Point Clamped inverter [11].

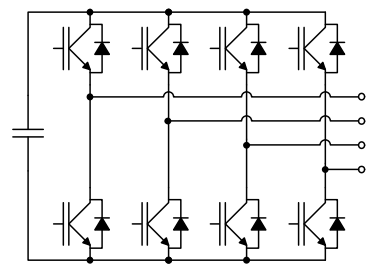

Figure 2. The four-legs inverter [12].

introduce additional switching and conduction losses, as a consequence the efficiency is lower than in the conventional three-phase inverter.

- In [14], the authors propose a solution where seven switches are present (shown in Fig. 4), allowing to break the leakage current conduction path during the freewheeling period. It utilizes RCMV-PWM for reducing the CMV through the elimination of the zero voltage states. However, it only focuses on leakage current elimination compromising issues such as voltage linearity, output current ripple, dc-link current ripple and harmonic distortion.

- The impedance-source converter, depicted in Fig. 5, adds to the H6 structure an impedance network on the source side, resulting in a buck-boost inverter. It can be applied

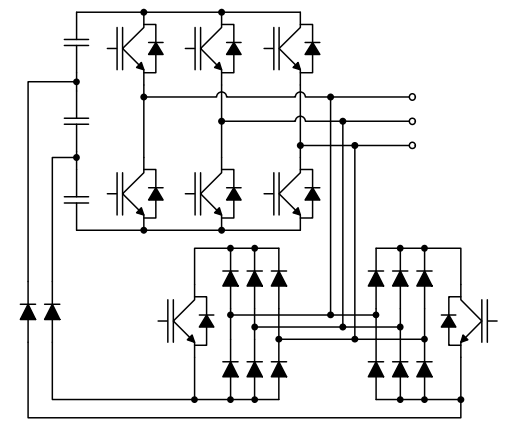

Figure 3. The Two-level Three-phase PV inverter topology [13].

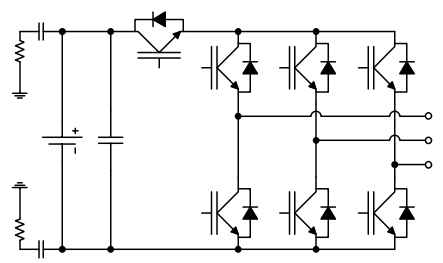

Figure 4. The H7 architecture [14].

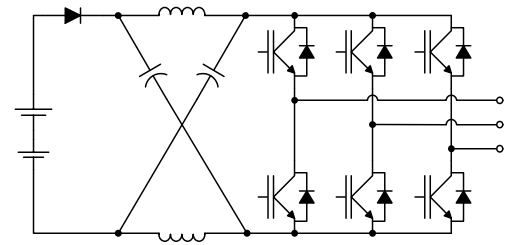

Figure 5. The Z-source inverter [15].

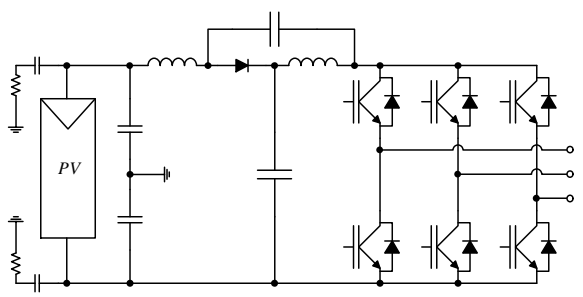

Figure 6. The Quasi-Z-source architecture [16].

to every power conversions and to adjustable speed drive, especially in applications where the input voltage changes widely. It exhibits good efficiency, can minimize stresses and size of the motor and performs a higher output power than a conventional PWM inverter. However, it is suitable for boost ratio range up to 2, while for higher ratios the DC/DC boosted PWM inverter is the best configuration. Furthermore, the presence of a right-hand-plane zero limits the dynamic response and it cannot be eliminated by adjusting the Z-source parameters [15], [19].

- The Quasi-Z-source inverter, depicted in Fig. 6, represent a modified topology with respect to the impedence-source inverter, with all the advantages of the ZSI and additional benefits such as a constant input current and less stress on components, moreover it is suitable for SVPWM modulations for minimizing the CMV [16].

- A topology called DCM-232 is depicted in Fig. 7. It makes use of ten switches, six for the H6 and four (unidirectional, with reverse blocking capability) for a DC multiplexer with two DC sources (PV panels). Both of them are disconnected from the DC bus when the zero vectors are generated, so that the stray capacitance of the panels remains at a constant value and no leakage current arises. The solution performs well in terms CMV and leakage current reduction, however it needs separate DC sources and it increases the complexity both of the hardware and of the control because of the presence of ten switches [17].

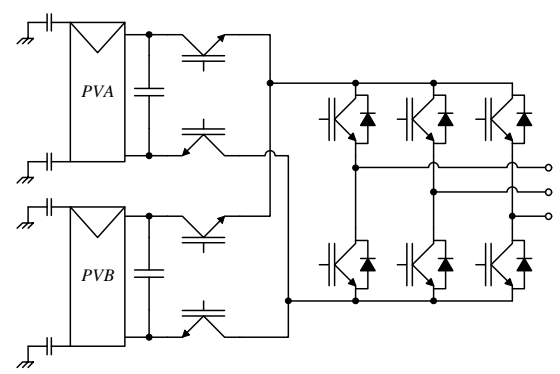

Figure 7. The DCM-232 inverter [17]. 


\section{Proposed InVERTER ARCHITECTURE AND MODULATION}

The proposed inverter architecture (shown in Fig. 8) differs from a traditional H6 for the addition of two active devices at the DC source side, a capacitor divider and two clamping diodes. Deriving from a H6 and having a total of eight active devices, the proposed inverter architecture has been named "H8". The two additional active devices, referred to as $T 7$ and $T 8$, are placed between the DC source and the three legs block, and they act as DC-decoupling devices during the current freewheeling phases. A capacitive divider is placed on the DC side of the inverter, allowing to obtain voltages equal to $\frac{1}{3} V_{D C}$ and $\frac{2}{3} V_{D C}$, i.e. the CMV values respectively during the active odd and even states. In order to ensure an equal partition of the voltage across the capacitors, an high impedance (to reduce the power losses) resistive divider was added in parallel. Two diodes, named $D_{H}$ and $D_{L}$, are placed between the two intermediate points of the divider and the H6 bridge in order to clamp the voltage at the high side or at the low side of the bridge itself, during the upper or bottom current freewheeling phases, depending on the case.

The CMV is defined as the average of the voltages between the inverter outputs and the negative DC source (addressed as $N)$ :

$$
V_{C M}=\frac{V_{u N}+V_{v N}+V_{w N}}{3}
$$

Table I summarizes the common mode voltage values at the inverter output, both for a traditional three-phase inverter and for the proposed $\mathrm{H} 8$ topology. In the case of a traditional $\mathrm{H} 6$, the CMV varies from 0 to $V_{D C}$, with a $\frac{1}{3} V_{D C}$ step at every state commutation. The $\mathrm{H} 8$ topology only presents two values for the CMV: $\frac{1}{3} V_{D C}$ for all the odd states and $\frac{2}{3} V_{D C}$ for all the even ones. In fact, differently from the H6, the proposed solution exhibits the same CMV values both during the active state and the inactive ones. This is possible for the presence of the DC-decoupling devices $\left(T_{7}\right.$ and $\left.T_{8}\right)$ and of the voltage-clamping diodes $\left(D_{H}\right.$ and $\left.D_{L}\right)$. Before the high side current freewheeling phase (state 8 , or vector $V_{8}$ ), when all the three high side devices in the bridge are on, the upper DC-decoupling device $\left(T_{7}\right)$ is switched off and this causes the diode $D_{H}$ to turn on clamping the voltage on the upper

Table I

COMMON-MOdE VOLTAGE $\left(V_{C M}\right)$ VALUES

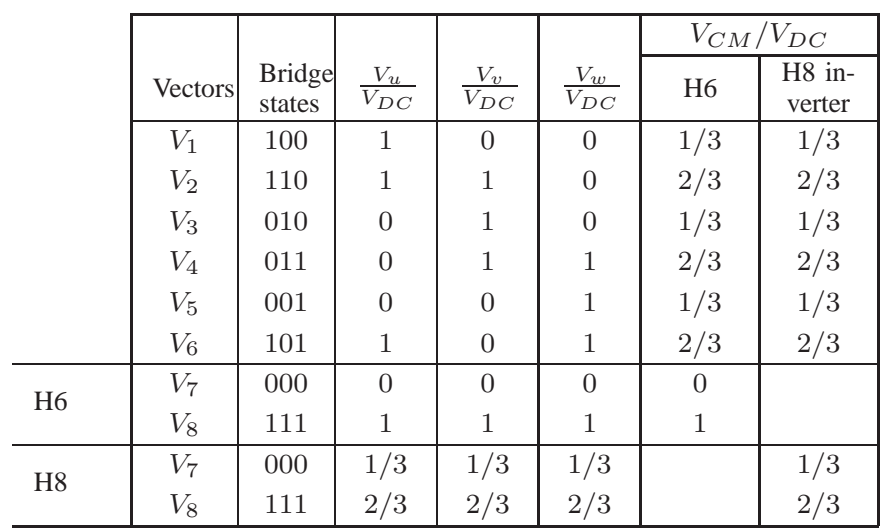

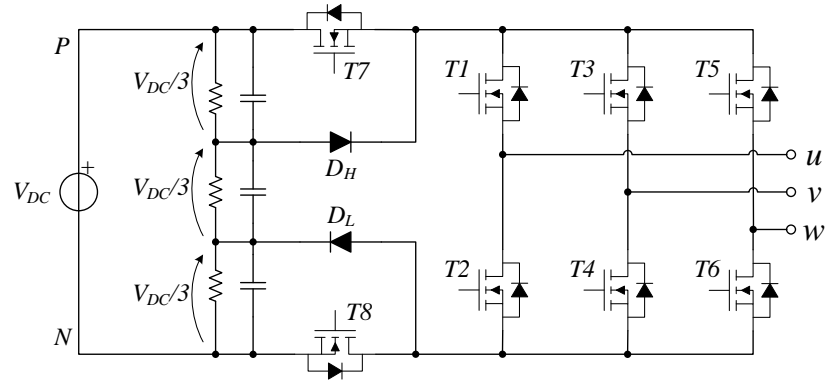

Figure 8. The proposed H8 inverter topology.

rail of the bridge to $\frac{2}{3} V_{D C}$. Similarly, before the low side current freewheeling phase (state 7 , or vector $V_{7}$ ), when all the three low side devices in the bridge are on, the bottom DC-decoupling device $\left(T_{8}\right)$ is switched off and this causes the diode $D_{L}$ to turn on and therefore the voltage on the bottom part of the bridge to be $\frac{1}{3} V_{D C}$. Then, for both cases, for the remaining duration of the inactive states the voltage on the interested side of the bridge maintains those values. Figure 9 shows the eight configurations of the inverter for the eight SV states.

Since the two clamping diodes ensure the voltage across $T_{7}$ and $T_{8}$ to be only one third of the total DC bus, devices with a reduced breakdown voltage can be adopted, reducing than the additional losses those devices introduce.

In order to force $D_{H}$ or $D_{L}$ to turn on when the inverter enters into the zero states, the decoupling devices turn off is anticipated with respect to the commutation of the bridge devices. For example, in the transition from state 1 to state 7, first $T_{8}$ is switched off, leading to $D_{L}$ to turn on (Fig. 10(a)); then $T_{1}$ is switched off and after a dead time $T_{2}$ is switched on, completing the state commutation. Fig. 10 shows the configurations of the inverter which are put in place at every transitions from one active states to the following inactive one. As a matter of fact, during these temporary phases $V_{C M}$ assumes one of the following two values:

$$
\begin{aligned}
& V_{C M}^{\prime}=\frac{1}{3}\left(1+\frac{1}{3}+\frac{1}{3}\right) V_{D C}=\frac{5}{9} V_{D C}=0.55 V_{D C} \\
& V_{C M}^{\prime \prime}=\frac{1}{3}\left(0+\frac{2}{3}+\frac{2}{3}\right) V_{D C}=\frac{4}{9} V_{D C}=0.44 V_{D C}
\end{aligned}
$$

In particular, $V_{C M}=V_{C M}^{\prime}$ in the cases involving odd vectors, depicted in Fig. 10(a), 10(c) and 10(e), whereas $V_{C M}=V_{C M}^{\prime \prime}$ in the cases involving even vectors, showed in Fig. 10(b), 10(d) and 10(f). This implies that CMV is not constant throughout a PWM period, but it varies from $\frac{1}{3} V_{D C}$ to $\frac{5}{9} V_{D C}$ (odd cases) and from $\frac{2}{3} V_{D C}$ to $\frac{4}{9} V_{D C}$ (even cases) at every transition from an active to an inactive vector. Nevertheless, these variations are smaller than those typical of traditional solutions. In particular, for the two cases they result to be:

$$
\begin{aligned}
& \Delta V_{C M}^{\prime}=\frac{5}{9} V_{D C}-\frac{1}{3} V_{D C}=+\frac{2}{9} V_{D C}=+0.22 V_{D C} \\
& \Delta V_{C M}^{\prime \prime}=\frac{4}{9} V_{D C}-\frac{2}{3} V_{D C}=-\frac{2}{9} V_{D C}=-0.22 V_{D C}
\end{aligned}
$$

It is worth to be noted that $D_{H}$ or $D_{L}$ turn on only for few nanoseconds, charging or discharging the stray capacitance of 


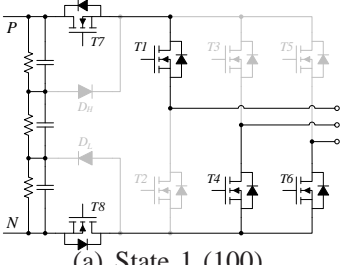

(a) State 1 (100).
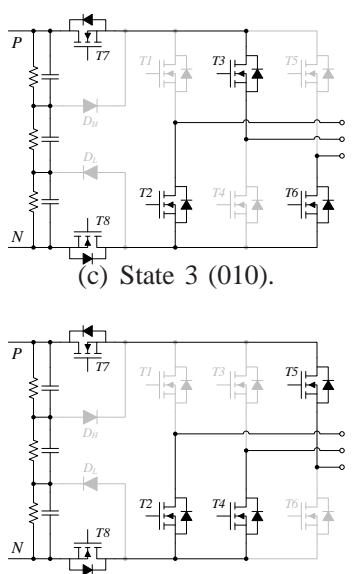

(e) State 5 (001).

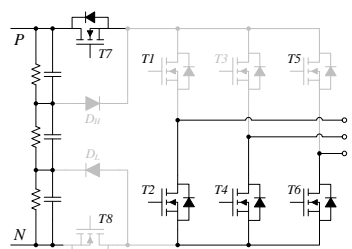

(g) State 7 (000).

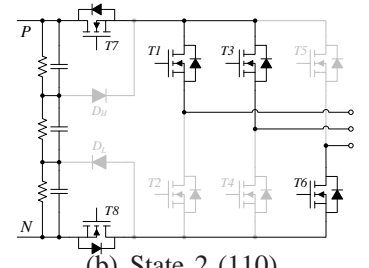

(b) State 2 (110).

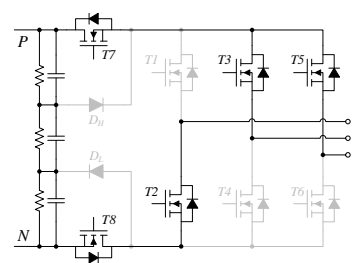

(d) State 4 (011).

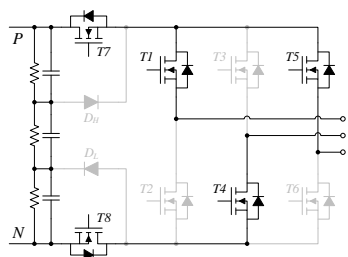

(f) State 6 (101).

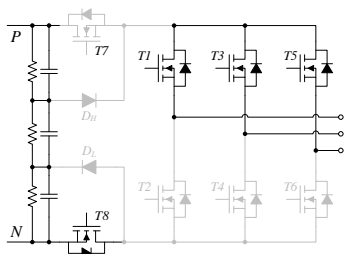

(h) State 8 (111).
Figure 9. Configurations of the H8 inverter for the eight Space Vector states. Devices are depicted in black if they are on, in gray if they are off. Current conduction through anti-parallel diodes are neglected for simplicity.

the devices, therefore not causing a significant unbalance in the voltage divider. However, they are passive devices, so they need a current flowing from the DC side to the AC side in order to turn on. In case of zero current, this does not occur, hence the CMV deteriorates.

The modulation adopted for the control of the inverter is a novel Space Vector (SV) strategy, specifically developed in conjunction with the H8 architecture, with the aim to achieve an almost constant CMV (as previously discussed, $\pm \frac{2}{9} V_{D C}$ variations are unavoidable) and performing single leg commutations. In the following, this strategy will be addressed as Constant Common-Mode Voltage Space Vector (CCMV$\mathrm{SV}$ ), however it can only be applied for modulation indexes lower than 0.5 (Fig. 13(b) and 13(c)). In order to overcome to the linearity range limitation, the system can switch to a different control strategy when a higher modulation index is needed. In such a case, a Near State Space Vector (NS-SV) is adopted, since this extends the modulation index range up to $\sqrt{3} / 2$ (see Fig. 13(d)). Compared to CCMV-SV, NS-SV performs worse in terms of CMV, but in conjunction with the proposed topology it ensures advantages over conventional three-phase inverters. The following two subsections discuss in further detail the two developed modulation strategies.

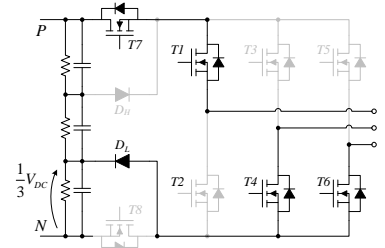

(a) Leading phase between state 1 and state 7 .

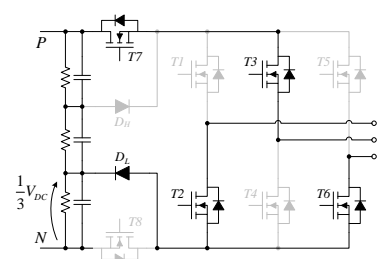

(c) Leading phase between state 3 and state 7.

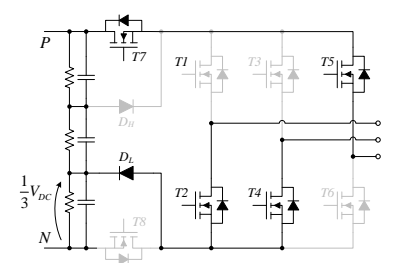

(e) Leading phase between state 5 and state 7 .

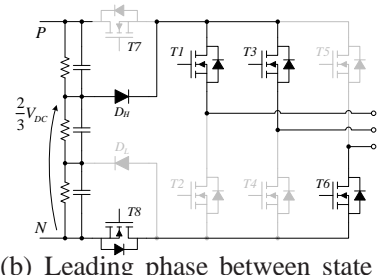

(b) Leading phase between state 2 and state 8

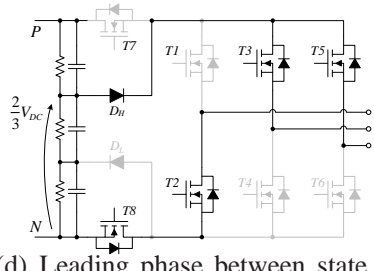

(d) Leading phase between state 4 and state 8.

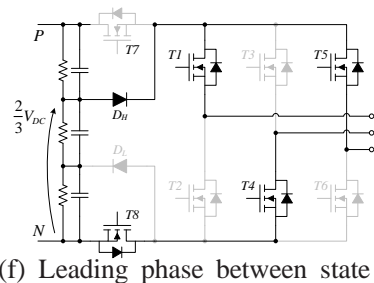

(f) Leading phase between state 6 and state 8
Figure 10. Configurations of the $\mathrm{H} 8$ inverter during transitions from active to inactive states. Devices are depicted in black if they are on, in gray if they are off. Current conduction through anti-parallel diodes are neglected for simplicity.

\section{A. Modulation index smaller than 0.5: CCMV-SV modulation}

In order to maintain a constant CMV at the inverter output, this SV strategy only makes use of those vectors exhibiting the same $V_{C M}$. Table I shows the ratios between $V_{C M}$ and $V_{D C}$ for all the eight $\mathrm{SV}$ states. As a matter of fact, odd active vectors $\left(V_{1}, V_{3}\right.$ and $\left.V_{5}\right)$ exhibit $V_{C M}=\frac{1}{3} V_{D C}$, while even active vectors $\left(V_{2}, V_{4}\right.$ and $\left.V_{6}\right)$ have $V_{C M}=\frac{2}{3} V_{D C}$. This is also true considering the $\mathrm{H} 6$ and, indeed, several modulations were proposed in literature attempting to keep common-mode voltage constant by using only a set of vectors that exhibit the same $V_{C M}$ [6]. Nevertheless, those modulations do not make use of inactive vectors $\left(V_{7}, V_{8}\right)$, and this results in simultaneous commutations of more than one bridge leg. On the contrary, adopting the $\mathrm{H} 8$ architecture, $V_{C M}$ values of the inactive vectors, thanks to the DC decoupling, can match them of the active states. In this context, it is possible to obtain the same CMV values with all the odd vectors $\left(V_{1}, V_{3}, V_{5}\right.$ and $\left.V_{7}\right)$, or with all the even ones $\left(V_{2}, V_{4}, V_{6}\right.$ and $\left.V_{8}\right)$. As already mentioned in Sec. III, at every transition from an active state to an inactive one, CMV becomes equal to $\frac{4}{9} V_{D C}$ or to $\frac{5}{9} V_{D C}$ (see Eq. 2 and 3), leading to a $\pm \frac{2}{9} V_{D C}$ variation of CMV.

The reference vector in the $\alpha-\beta$ plane is synthesized by the use of the two nearest odd or even vectors with the proper modulated duration, plus the proper inactive state fulfilling the remaining time in the PWM period. Applying this scheme, the points which can be synthesized are included in two equilateral triangles having vertexes on the applied active vectors (see Fig. 13(b) and 13(c)). The maximum modulation index which 
Table II

VECTORS US AGE IN CCMV-SV MODULATION STRATEGY

\begin{tabular}{|c|c|c|}
\hline Angles & Odd vectors & Even vectors \\
\hline $0^{\circ}-60^{\circ}$ & \multirow{2}{*}{$V_{1}, V_{3}, V_{7}$} & $V_{2}, V_{6}, V_{8}$ \\
\cline { 1 - 1 } & & $V_{2}, V_{4}, V_{8}$ \\
\hline $60^{\circ}-120^{\circ}$ & & \\
\cline { 1 - 1 } $120^{\circ}-180^{\circ}$ & \multirow{2}{*}{$V_{5}, V_{7}$} & $V_{4}, V_{6}, V_{8}$ \\
\cline { 1 - 1 } $180^{\circ}-240^{\circ}$ & & \\
\cline { 1 - 1 } $240^{\circ}-300^{\circ}$ & \multirow{2}{*}{$V_{5}, V_{1}, V_{7}$} & $V_{2}, V_{6}, V_{8}$ \\
\hline $300^{\circ}-360^{\circ}$ & & \\
& &
\end{tabular}

is possible to maintain throughout the entire rotational period is 0.5 . This is a rather limiting feature for an electric drive, therefore a different modulation strategy has been adopted for modulation indexes greater than 0.5 (NS-SV), which is exposed in subsection III-B.

In order to perform a single leg commutation for each state transition, the $\alpha-\beta$ plane has been divided into different zones, each one featuring different state sequences. Considering the odd vectors case, shown in Fig. 13(b), there are six $60^{\circ}$-wide zones (nr. 1, 3, 4, 6, 7 and 9), plus 3 zones without a specific angular width, represented as very narrow slices (nr. 2, 5 and 8). Such narrow zones are intended to represent SV sequences just applied for a single PWM period, because they are necessary for matching the state sequences performed in their two adjacent zones. A similar subdivision exists for the dual case adopting even vectors, as shown in Fig. 13(c). Table II summarizes the vectors usage for all the zones throughout the entire rotation period, for odd and even CCMV-SV strategy. In order to achieve symmetry during the switching period, in the $60^{\circ}$-wide zones one of the two active states is split into two separated slots of time at the margin of the sequences. The pulse pattern during a switching period is shown in Fig. 11 for all the zones in the $\alpha-\beta$ plane, both in case of even and odd vectors. In case of no $60^{\circ}$ wide zones $(2,5,8,11,14$ and 17) the symmetry is not maintained because, for their native purpose, in those cases sequences must begin and finish with different active states. Moreover, the location of these zones in the $\alpha-\beta$ plane implies that the two modulation values for the active states will be approximately equal. Featuring only three states instead of five (see the central portions of Fig. 11), these intermediate slots must last half the time than the others, otherwise the output would produce a bigger current ripple.

\section{B. Modulation index greater than 0.5: NS-SV modulation}

Whenever the control loop requires for the inverter a modulation index higher than 0.5 , the control function automatically changes the NS-SV, allowing the inverter to work up to a modulation index equal to $\sqrt{3} / 2$. This strategy makes use of the two adjacent active vectors on the $\alpha-\beta$ plane (hence the name Near State modulation), and both the two inactive vectors. States sequences have been designed in order to ensure single leg commutations throughout all the $360^{\circ}$; the resulting pulse pattern is shown in Fig. 12. During each PWM period the time dedicated to inactive vectors is equally distributed between both $V_{7}$ and $V_{8}$; sequences exhibit a central symmetry

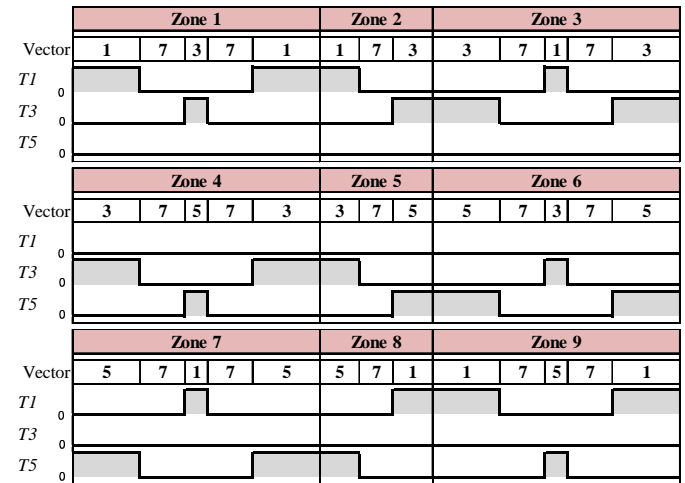

(a)

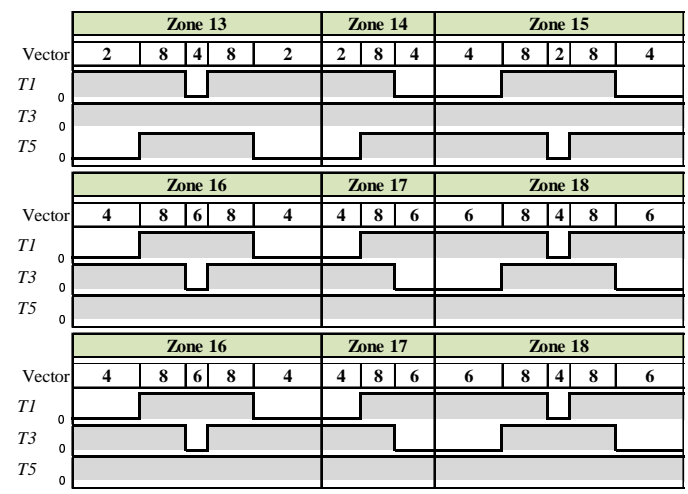

(b)

Figure 11. Vectors usage and switch pulse pattern throughout the rotational period for odd vectors CCMV-SV (a) and even vectors CCMV-SV (b). For each zone only one PWM period is represented. Widths of the time slots are chosen as case of example.

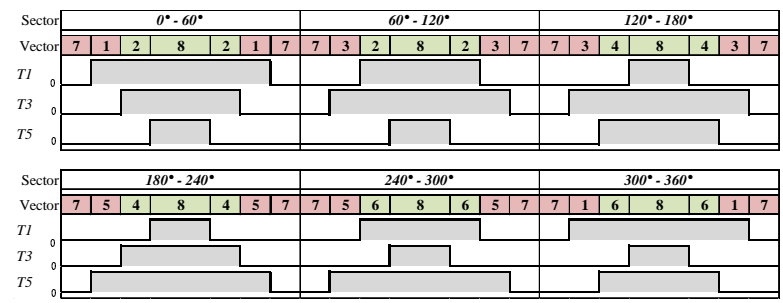

Figure 12. Vectors usage and switch pulse pattern throughout the rotational period for the NS-SV modulation strategy. For each angular sector only one PWM period is represented. Odd vectors are highlighted in red, even vectors in green. Widths of the time slots are chosen as case of example.

and active states are put in place according to the minimum difference (in terms of switching configuration) they have with respect to the previous or subsequent inactive state. Similarly to the CCMV-SV, $V_{C M}$ becomes equal to $\frac{1}{3} V_{D C}$ when odd vectors are performed and $\frac{2}{3} V_{D C}$ when even vectors are. In each PWM period both odd and even vectors are exploited, so NS-SV exhibits a CMV varying between those two values at the PWM carrier frequency. Hence, compared to CCMV-SV more common-mode voltage is present.

\section{Simulation RESUlts}

A Matlab/Simulink model of the inverter has been designed, allowing to develop and test the system features. As the purpose of this work is to present the novel inverter architecture along with the proposed modulation strategies, it was 
(a) SV vectors diagram.

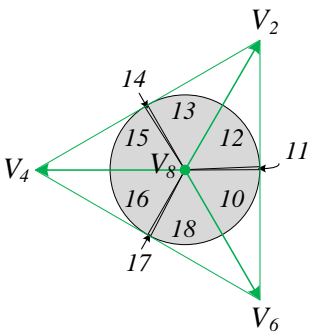

(c) Linearity region $(m<0.5)$ and modulation zones numbering for even vectors CCMV-SV.

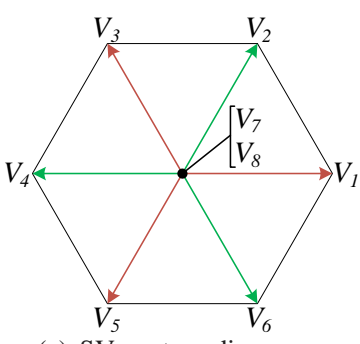

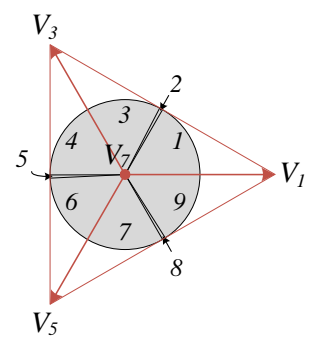

(b) Linearity region $(m<0.5)$ and modulation zones numbering for odd vectors CCMV-SV.

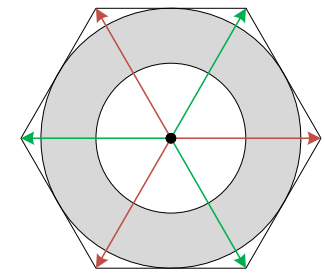

(d) The region where NS-SV is used $(0.5 \leq m<\sqrt{3} / 2)$.
Figure 13. Space Vector diagrams in the $\alpha-\beta$ plane. Odd vectors are red colored, even vectors are green colored.

introduced a RL load in wye connection, with floating neutral point. The main parameters used for the simulations are given in Tab. III.

In Fig. 14, $V_{C M}$ is shown for three cases of comparison: H6 with NS-SV, H8 with NS-SV and H8 with CCMV-SV. The H6 exhibits a $V_{C M}$ varying from 0 to $V_{D C}$ at the PWM frequency, the H8 inverter with NS-SV halves the number of $V_{C M}$ transitions and limits its excursion to $\frac{1}{3} V_{D C}-\frac{2}{3} V_{D C}$, whereas the H8 inverter with CCMV-SV performs an almost constant $V_{C M}$. For the last two cases, adopting $\mathrm{H} 8$ architecture, CMV exhibits spikes at $\frac{4}{9} V_{D C}$ or $\frac{5}{9} V_{D C}$, depending on the odd or even vectors usage. These spikes occur in the transitions from active to inactive states, as discussed in Sec. III. For this comparison, a modulation index $m=0.5$ was adopted for all the cases.

In Fig. 15 the output currents for the $\mathrm{H} 8$ architecture with CCMV-SV modulation and a modulation index $m=0.4$ are depicted. It is worth to be noted that before $0.02 \mathrm{~s}$ the modulation makes use of odd vectors, causing the current ripple to be greater in the upper part of the sinusoid, whereas after $0.02 \mathrm{~s}$ the modulation changes to the usage of even vectors, causing an opposite behavior of the ripple.

Table III

SIMULATION PARAMETERS

\begin{tabular}{lc}
\hline Parameter & Value \\
\hline$V_{D C}$ & $600 \mathrm{~V}$ \\
$f_{S W}$ & $10 \mathrm{kHz}$ \\
$f_{e}$ & $50 \mathrm{~Hz}$ \\
Wye load & $3 \times(10 \Omega+2 \mathrm{mH})$ \\
\hline
\end{tabular}

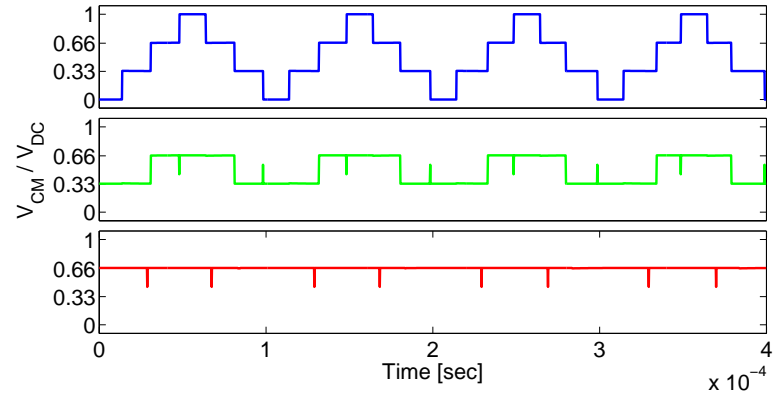

Figure 14. CMV evolution over four PWM periods in case of the inverter performing H6 with NS-SV (upper), H8 with NS-SV (central) and H8 with even-CCMV-SV (bottom).

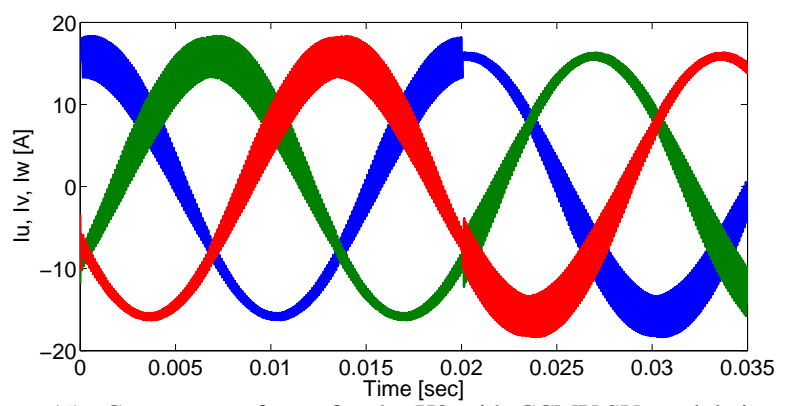

Figure 15. Current waveforms for the H8 with CCMV-SV modulation.

The spectrum of the output voltage was also investigated and the harmonic content of the common and differential voltages are depicted in Fig. 16 and Fig. 17, where the amplitudes of harmonics are normalized respectively to $V_{D C}$ and to the amplitude of the fundamental. It is possible to see how the harmonic content of the CMV is reduced for CCMV-SV over NS-SV modulation of about four decades, while the spectrum of the differential voltage is substantially the same for all the three examined cases. For this comparison, all simulations were conducted with $m=0.4$ and for a simulation time $t=0.5 \mathrm{~s}$, hence lasting 25 fundamental cycles.

A drawback of the proposed modulation consists in the increased distortion of the output voltage. This was investigated evaluating the weighted total harmonic distortion (WTHD), which is expressed as [20]:

$$
W T H D=\frac{\sqrt{\sum_{n=2}^{\infty}\left(\frac{V_{n}}{n}\right)^{2}}}{V_{1}}
$$

Figure 18 reports the WTHD for the two proposed modulations against the modulation index. As expected, CCMV-SV

Table IV

COMPARISON OF H8 WITH DIFFERENT MODULATIONS AND H6

\begin{tabular}{lccc}
\hline Topology & H6 & H8 & H8 \\
Modulation & NS-SV & NS-SV & CCMV-SV \\
\hline DC clamping & No & Yes & Yes \\
Maximum mod. index & 1 & 0.866 & 0.5 \\
CMV & High & Medium & Low \\
WTHD \% & $0.5-1.25$ & $0.5-1.25$ & $0.75-2.25$ \\
Efficiency & Medium & Low & High \\
\hline
\end{tabular}



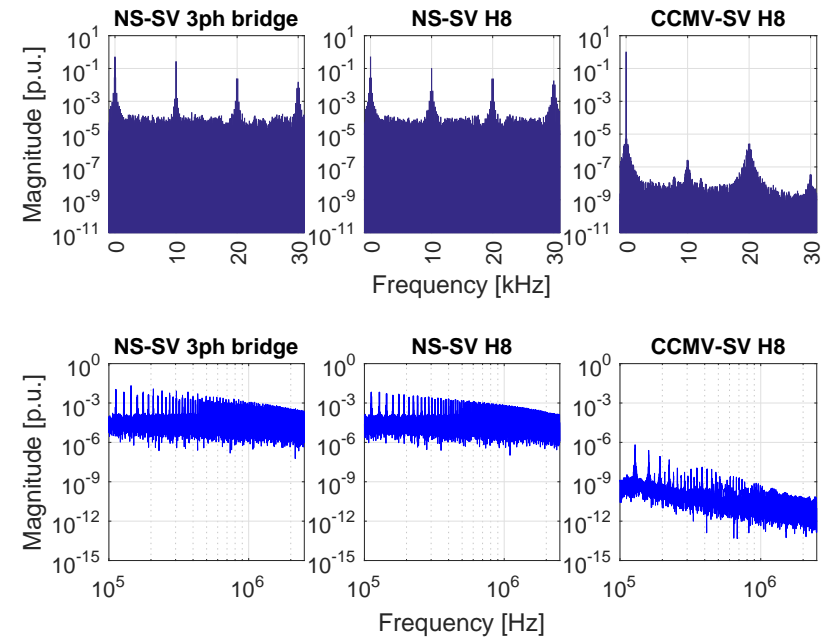

Figure 16. FFT of the CMV normalized to the supply voltage.

modulation exhibits a bigger output distortion than the NS-SV, resulting in a WTHD from $30 \%$ to $80 \%$ higher, depending on the modulation index. On the other hand, NS-SV modulation exhibit the same WTHD both in the case of the H6 and the $\mathrm{H} 8$ inverter.

As already mentioned in Section III, the proposed H8 has two additional devices for the decoupling of the DC side, which are responsible for additional losses. The efficiency of the proposed system was investigated and compared to a traditional one. Electrothermal simulations were conducted featuring the switching and conduction losses of a commercial power IGBT (Infineon IKW50N65H5). Figure 19 illustrates the performance for different combinations of the system in terms of topology and modulation (H8 or H6 and CCMV$\mathrm{SV}$ or NS-SV). In particular, comparing the cases relative to the NS-SV modulation with $\mathrm{H} 8$ and $\mathrm{H} 6$ allows to evaluate the losses arising from the additional devices on the DC side of the H8 topology: the efficiency detriment results about $0.45 \%$. Furthermore, comparing the cases with the same H8 configuration and the two proposed modulations, it is possible to observe that CCMV-SV performs better than NS-SV, with an efficiency improvement from $0.3 \%$ to $1.75 \%$ depending on the modulation index.

Table V

EXPERIMENTAL TEST CASES

\begin{tabular}{lccc}
\hline Test case & 1 & 2 & 3 \\
\hline Topology & H6 & H8 & H8 \\
DC clamping & No & Yes & Yes \\
Modulation & NS-SV & NS-SV & CCMV-SV \\
Mod. index $m$ & 0.6 & 0.6 & 0.25 \\
$V_{D C}$ & $250 \mathrm{~V}$ & $250 \mathrm{~V}$ & $500 \mathrm{~V}$ \\
$f_{S W}$ & \multicolumn{4}{c}{$5 \mathrm{kHz}$} \\
$f_{e}$ & $50 \mathrm{~Hz}$ \\
Load & $3 \times(10 \Omega+2 m H)$, wye connected \\
\hline
\end{tabular}
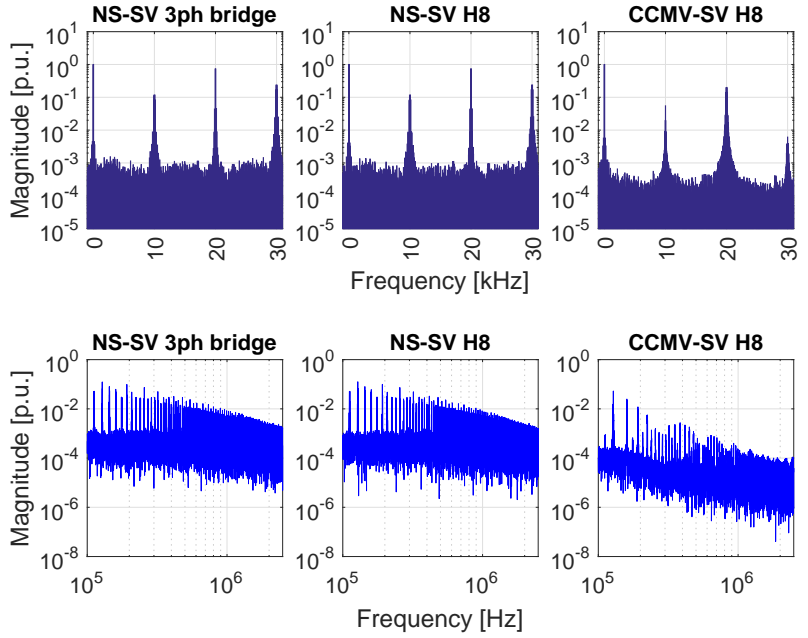

Figure 17. FFT of the line-to-line voltage normalized to the fundamental.

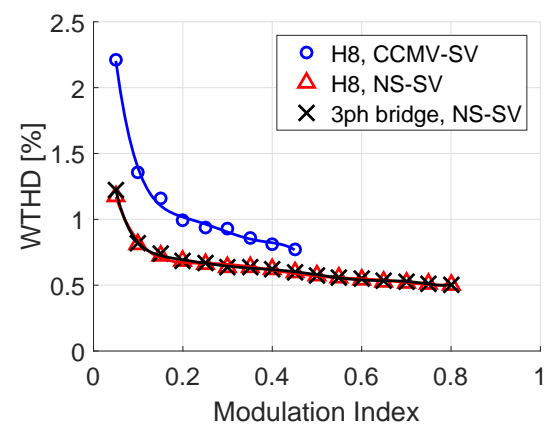

Figure 18. WTHD evolution against the modulation index for different configurations of the system.

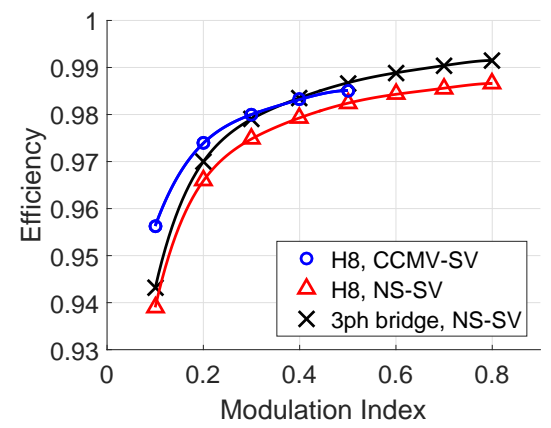

Figure 19. Efficiency performance for different combinations of the system.

\section{EXPERIMENTAL RESULTS}

In order to prove the effectiveness of the H8 architecture and the proposed modulation strategies, a preliminary inverter prototype was built. A logic board, featuring a Freescale MCU MPC5643L, performs the proposed modulation and the current control. Test bed included a wye connected RL load in accordance to the simulations.

In order to compare the proposed solution with a traditional one, the following three operational configurations of the system, also summarized in Tab. V, were tested.

1) Operating as a H6 (maintaining the two DC side devices always on, therefore bypassing the DC-decoupling 


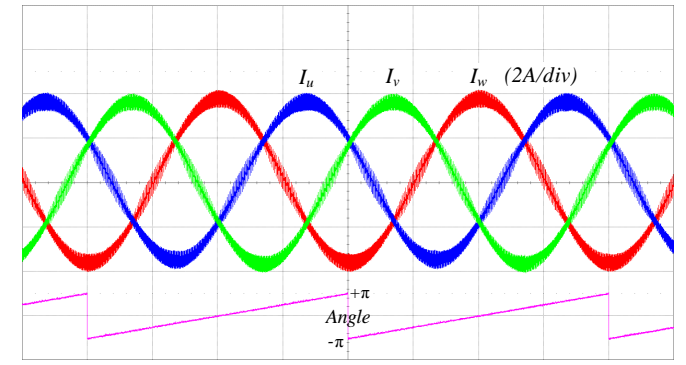

(a)

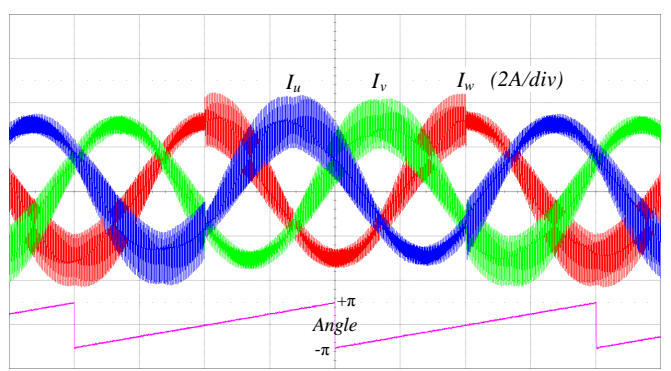

(b)

Figure 20. Current waveforms for the H8 prototype (blue, green and red) and evolution of the rotational angle (magenta) for two test cases: (a) NS-SV, $V_{D C}=250 \mathrm{~V}, m=0.6$; (b) CCMV-SV, $V_{D C}=500 \mathrm{~V}, m=0.25$. Time scale is $5 \mathrm{~ms} / \mathrm{div}$.

feature of the $\mathrm{H} 8$ architecture) and performing NS-SV modulation.

2) Enabling the DC decoupling during the inactive states execution and adopting the NS-SV modulation.

3) Enabling the DC decoupling during the inactive states execution and adopting the CCMV-SV modulation.

Tests present results in agreement with simulations. Fig. 20 presents current waveforms both in case of $\mathrm{H} 8$ performing NS-SV modulation, with a fixed modulation index of 0.6 , and in case of H8 with CCMV-SV modulation with a fixed modulation index of 0.25 . In the latter, the change in the current ripple behavior at every zero-crossing of the rotational angle is caused by the CCMV-SV modulation changing from odd to even vectors and vice versa. Moreover, in the CCMVSV case the current ripple results to be higher than the NS-SV case. This is caused by two factors: CCMV-SV performs worse than NS-SV regarding this aspect, DC source voltage in the second testbed was the double than that in the first one.

Fig. 21 shows the reduction in $V_{C M}$ for $\mathrm{H} 8$ over $\mathrm{H} 6$ and for CCMV-SV over NS-SV modulation. For the H6 $V_{C M}$ varies in the range from $0 V$ to $V_{D C}$ at the PWM frequency (Fig. 21(a)); for H8 with NS-SV modulation the excursion of $V_{C M}$ is reduced to $\frac{1}{3} V_{D C}-\frac{2}{3} V_{D C}$ (Fig. 21(b)); finally $\mathrm{H} 8$ with CCMV-SV modulation performs $V_{C M}$ varying between $\frac{1}{3} V_{D C}$ and $\frac{2}{3} V_{D C}$ only once every rotational period, when the system changes between odd and even vectors (Fig. 21(c)), and exhibiting only small spikes around those main levels.

\section{CONCLUSIONS}

In this work a modified two-level inverter was developed to reduce the common mode voltage. It makes use of a novel architecture called $\mathrm{H} 8$, which adds at the DC side of

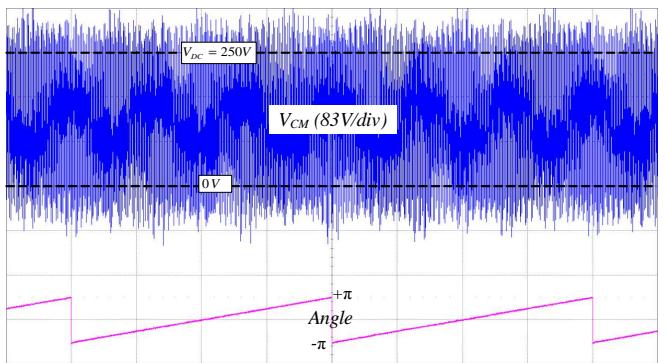

(a)

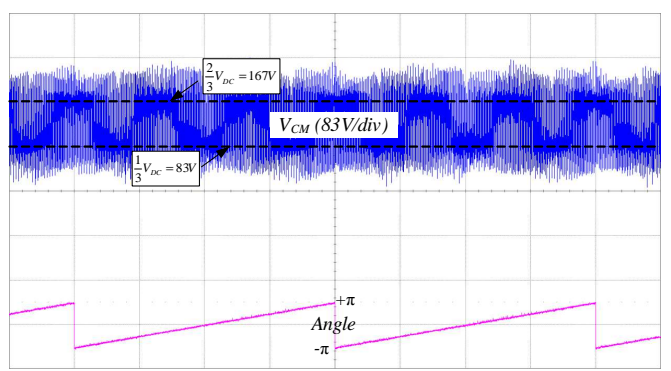

(b)

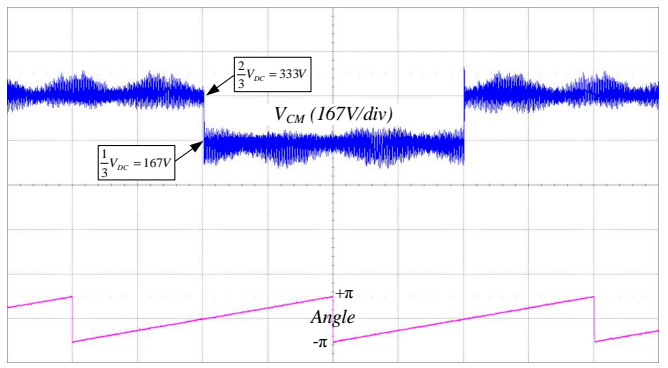

(c)

Figure 21. Common-mode voltage and rotational angle evolution for (a) the H6 performing NS-SV, $V_{D C}=250 \mathrm{~V}$; (b) H8 performing NS-SV, $V_{D C}=$ $250 \mathrm{~V}$; (c) $\mathrm{H} 8$ performing CCMV-SV $V_{D C}=500 \mathrm{~V}$. Time scale is $5 \mathrm{~ms} / \mathrm{div}$.

a conventional H6 two active devices for decoupling the DC source and a passive circuit able to fix the voltage of the bridge at a reference value during the freewheeling phases. Moreover, two PWM modulation strategies have been developed as customized Space Vector modulations. One, denoted as CCMV-SV, is able to maintain the $V_{C M}$ constant for an entire rotational period in case of modulation index smaller than 0.5. For higher modulation index, the control function exploits a different modulation strategy (NS-SV) that ensures a wider modulation range for the system at price of increased $V_{C M}$. Simulations have been conducted on Matlab/Simulink environment with good results. A preliminary prototype has been built and experimental results obtained, showing good performance of the system, in accordance with computer simulations. The proposed solutions allows reducing commonmode voltage both in amplitude and frequency respect to the two-level inverter.

\section{REFERENCES}

[1] D. Rendusara and P. Enjeti, "An improved inverter output filter configuration reduces common and differential modes $\mathrm{dv} / \mathrm{dt}$ at the motor terminals in pwm drive systems," IEEE Transactions on Power Electronics, vol. 13, no. 6, pp. 1135-1143, Nov 1998. 
[2] D. Boillat, J. Kolar, and J. Muhlethaler, "Volume minimization of the main $\mathrm{dm} / \mathrm{cm}$ emi filter stage of a bidirectional three-phase three-level pwm rectifier system," in Energy Conversion Congress and Exposition (ECCE), 2013 IEEE, Sept 2013, pp. 2008-2019.

[3] C. Choochuan, "A survey of output filter topologies to minimize the impact of pwm inverter waveforms on three-phase ac induction motors," in Power Engineering Conference, 2005. IPEC 2005. The 7th International, Nov 2005, pp. 1-544.

[4] N. Hensgens, M. Silva, J. Oliver, P. Alou, O. Garcia, and J. Cobos, "Analysis and optimized design of a distributed multi-stage emc filter for an interleaved three-phase pwm-rectifier system for aircraft applications," in Applied Power Electronics Conference and Exposition (APEC), 2012 Twenty-Seventh Annual IEEE, Feb 2012, pp. 465-470.

[5] L. Concari, D. Barater, C. Concar, and G. Buticchi, "A novel threephase inverter for common-mode voltage reduction in electric drives," in Energy Conversion Congress and Exposition (ECCE), 2015 IEEE, Sept 2015, pp. 2980-2987.

[6] A. Hava and E. Un, "A high-performance pwm algorithm for commonmode voltage reduction in three-phase voltage source inverters," IEEE Transactions on Industrial Electronics, vol. 26, no. 7, pp. 1998-2008, July 2011

[7] K. Li, T. Lu, Z. Zhao, L. Yin, F. Liu, and L. Yuan, "Carrier based implementation of reduced common mode voltage pwm strategies," in ECCE Asia Downunder (ECCE Asia), 2013 IEEE, 2013, pp. 578-584.

[8] A. Hava and E. Un, "Performance analysis of reduced common-mode voltage pwm methods and comparison with standard pwm methods for three-phase voltage-source inverters," IEEE Transactions on Power Electronics, vol. 24, no. 1, pp. 241-252, 2009.

[9] F. Zare, J. Adabi, A. Nami, and A. Ghosh, "Common mode voltage in a motor drive system with pfc," in Power Electronics and Motion Control Conference (EPE/PEMC), 2010 14th International, 2010, pp. T4-57-T4-64.

[10] H.-D. Lee and S.-K. Sul, "Common-mode voltage reduction method modifying the distribution of zero-voltage vector in pwm converter/inverter system," IEEE Transactions on Industry Applications, vol. 37, no. 6, pp. 1732-1738, 2001.

[11] A. Nabae, I. Takahashi, and H. Akagi, "A new neutral-point-clamped pwm inverter," IEEE Transactions on Industry Applications, vol. IA-17, no. 5, pp. 518-523, 1981.

[12] G. Oriti, A. Julian, and T. Lipo, "A new space vector modulation strategy for common mode voltage reduction [in pwm invertors]," in Power Electronics Specialists Conference, 1997. PESC '97 Record., 28th Annual IEEE, vol. 2, 1997, pp. 1541-1546 vol.2.

[13] G. Vazquez, T. Kerekes, J. Rocabert, P. Rodriguez, R. Teodorescu, and D. Aguilar, "A photovoltaic three-phase topology to reduce common mode voltage," in Industrial Electronics (ISIE), 2010 IEEE International Symposium on, 2010, pp. 2885-2890.

[14] K. Tan, N. Rahim, W.-P. Hew, and H. S. Che, "Modulation techniques to reduce leakage current in three-phase transformerless h7 photovoltaic inverter," IEEE Transactions on Industrial Electronics, vol. 62, no. 1, pp. 322-331, 2015.

[15] F. Bradaschia, M. Cavalcanti, P. Ferraz, and F. Neves, "Modulation for three-phase transformerless z-source inverter to reduce leakage currents in photovoltaic systems," IEEE Transactions on Industrial Electronics, vol. 58, no. 12, pp. 5385-5395, 2011.

[16] Y. Siwakoti and G. Town, "Three-phase transformerless grid connected quasi z-source inverter for solar photovoltaic systems with minimal leakage current," in Power Electronics for Distributed Generation Systems (PEDG), 2012 3rd IEEE International Symposium on, 2012, pp. 368373

[17] P. Rodriguez, R. Munoz-Aguilar, G. Vazquez, I. Candela, E. Aldabas, and I. Etxeberria-Otadui, "Symmetrical ripple constant common mode voltage modulation strategy for dcm-232 three-phase pv topology," in IECON 2011 - 37th Annual Conference on IEEE Industrial Electronics Society, 2011, pp. 2505-2510.

[18] M. Cavalcanti, K. de Oliveira, A. de Farias, F. Neves, G. Azevedo, and F. Camboim, "Modulation techniques to eliminate leakage currents in transformerless three-phase photovoltaic systems," IEEE Transactions on Industrial Electronics, vol. 57, no. 4, pp. 1360-1368, 2010.

[19] A. Florescu, O. Stocklosa, M. Teodorescu, C. Radoi, D. Stoichescu, and S. Rosu, "The advantages, limitations and disadvantages of z-source inverter," in Semiconductor Conference (CAS), 2010 International, vol. 02, 2010, pp. 483-486.

[20] D. G. H. T. A. Lipo, Pulse Width Modulation for Power Converters: Principles and Practice. Wiley-IEEE Press, 2003. [Online]. Available: http://ieeexplore.ieee.org/xpl/bkabstractplus.jsp?bkn=5264450

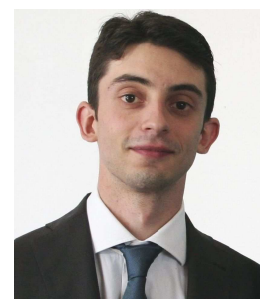

Luca Concari (S'14) was born in Parma, Italy, in 1988. He received the M.S. degree in Electronic Engineering in 2013 from the University of Parma, Italy. In 2014 he started the Ph.D. course in Information Technologies at the same University. During 2015 he was guest researcher at the Chair of Power Electronics, University of Kiel, Germany. His research area is focused on power electronics for static energy conversion and motor drives.

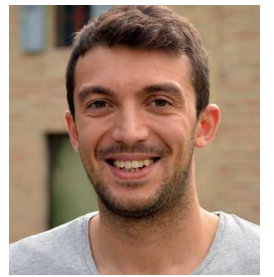

Davide Barater (S'11-M'14) was born in Italy in 1983. He received the M.S degree in Electronic Engineering in 2009 and the Ph.D. in Information Technologies in 2014 from the University of Parma, Italy. He was honorary scholar at the University of Nottingham, U.K, during 2012. He works as research fellow at the Department of Information Engineering, University of Parma. His research focuses on power electronics for renewable energy and motor drives. He is author or coauthor of more than 20 international papers and holds an international

patent.

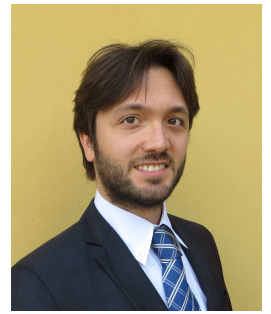

Giampaolo Buticchi (S'10-M'13) was born in Parma, Italy, in 1985. He received the Masters degree in Electronic Engineering in 2009 and the Ph.D degree in Information Technologies in 2013 from the University of Parma, Italy. He is now working as a postdoctoral research associate at the University of Kiel, Germany. His research area is focused on power electronics for renewable energy systems, smart transformer fed micro-grids and reliability in power electronics.

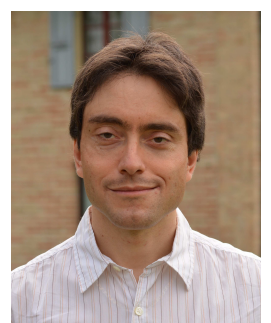

Carlo Concari (S'98-M'06) was born near Parma, Italy, in 1976. He received the M.S. degree in electronics engineering and the Ph.D. degree in information technology from the University of Parma, Parma, Italy, in 2002 and 2006, respectively. Since 2014 he is an Associate Professor with the Department of Information Engineering, University of Parma. His research activity is mainly focused on power electronics, digital drive control, static power converters, and electric machines diagnostics. He is the author or coauthor of more than 50 international

papers.

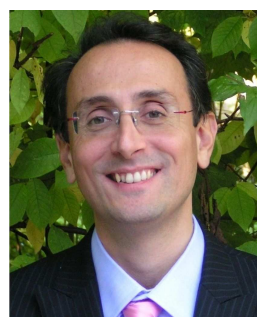

Marco Liserre (S'00-M'02-SM'07-F13) is Full Professor and Head of the Chair of Power Electronics at the University of Kiel (Germany). He has published over 200 technical papers and a book, for them he is listed in ISI Thomson report The worlds most influential scientific minds. He has been awarded with an European ERC Consolidator Grant, one of the most prestigious in Europe, to make research about Smart Transformer. He did serve IAS, PELS, PES and IES in various capacities such as reviewer, associate editor, editor, conference chairman or track chairman and Vice-President. He has received several IEEE Awards. 